\title{
EFFECTS OF WORKING CAPITAL MANAGEMENT ON FIRM PERFORMANCE AND FIRM VALUE - A STUDY OF THE FISHERIES INDUSTRY IN VIETNAM
}

\author{
VO THI QUY \\ International University - Vietnam National University HCMC - vtquy@ hcmiu.edu.vn \\ LE THI MINH NGUYEN \\ Van Hien University, Vietnam - minhnguyen50@ gmail.com
}

(Received: July 29, 2017; Revised: October 01, 2017; Accepted: October 31, 2017)

\begin{abstract}
This research investigates the effects of working capital management through cash conversion cycle and its components (average receivable days - ARD, average inventory days - AID, and average payable days - APD), along with the effects of the working capital management policies on firm performance and firm value in the fisheries industry. Generalized Method of Moment (GMM) was applied with the data collected from 21 fisheries companies listed on Vietnam's stock market in the period 2008 -2012. The research found that cash conversion cycle, average receivable days, average inventory days, and average payable days have a negative impact on firm performance (ROA) and firm value (Tobin'Q). The research results also showed that aggressive working capital policy has a negative impact, but conservative working capital policy has a positive impact on firm performance and firm value of fisheries selected companies.
\end{abstract}

Keywords: Cash conversion cycle; Fisheries industry; Vietnam stock market; Working capital Management.

\section{Introduction}

Fishery is a key economic sector of Vietnam, one of the top five economic sectors contributing significantly to the export performance of the country. Fisheries firms spend a huge amount of money to purchase raw materials for processing fishery products and storage seafood for exporting. In addition, with the characteristics of seasonal business and sales deferred, fisheries firms' current assets account for a large proportion of firm total assets.

In the past few years, due to the recession of world economy, the demand of seafood has also reduced; as a result the proportion of export has declined as well. In the context, many Vietnamese companies in the fishery sector stopped running business or even went to bankruptcy due to lack of working capital. This has indicated the importance of working capital management and its influence on the efficiency of business operations.

Currently, there are many studies related to management of working capital and firm's operation efficiency. However, lack of studies of the relationship between working capital management and firm efficiency in fisheries industry. This paper aims to: (1) determine the relationship between working capital management with fisheries firm performance and value, (2) measure the impact of working capital management components on firm performance and value of fisheries firms. The next sections will be literature review, data collection, research findings, and recommendation.

\section{Literature review and research model}

2.1. Definitions of working capital management

According to Ngo, T and Le, N (2015), working capital or gross working capital is the sum of short-term assets of a firm including 
cash and cash equivalents, short-term financial investments, accounts receivable, inventories, other short-term assets (prepaid expenses) used to finance the daily production and business activities.

Net working capital (NWC) is the difference between current assets and total short-term liabilities including short-term loans, account payables, accruals, and the other short-term liabilities.

Net operating working capital (NOWC) is the difference between operating current assets and operating current liabilities. Operating current assets include cash, accounts receivable, inventory, but excluding short-term financial investments. Operating current liabilities consist of accruals and account payables.

\subsection{Working capital management policies}

According to Moyer and CTG (1992), Eugene and Joel (2007), working capital is managed through two polices which are working capital investment policy and working capital financing policy.

Working capital investment policy (WCIP): According to Eugene and Joel (2007), the investment policy of working capital refers to the level of holding shortterm assets. There are three investment policies levels of investment in short-term assets as follows:

Applying conservative working capital investment policy a firm will hold a high level of cash and inventory. This policy helps a firm avoid the shortage costs but it increases the firm's financial costs. For aggressive working capital investment policy, a firm will invest in current assets at minimum level (Eugene and Joel, 2007). This policy reduces a firm's financial costs but it may increase shortage costs. Contrary to conservative investment, the policy is a risky policy, because company can face potential liquidity issues (Vahid and CTG, 2012).

Moderate working capital investment policy is seen as a combination in balance between conservative investment policy and aggressive working capital investment policy. This policy is the best policy standing balance between both angles of profits and risks.

(WCFP)

Working Capital Financing policy

According to Eugene and Joel (2007), Firer et al. (2008), investment in current assets or working capital needs to be financed from the sources of funds. The primary sources of capital for the working capital including bank loans, trade credit, prepaid expenses, longterm debts and owners' equity. Each source of capitals has its own advantages or disadvantages. Therefore, firms should make wise decisions on their best source of capital. Working capital financial policy is measured by the use of short-term debts, and it is distinguished through three financial policies as follows:

Conservative financial policy:

Firms use more long-term funds (including long-term debt and equity) to finance all long-term assets, permanent current asset and little of the needs of temporary working capital (seasonal or unexpected increasing of inventory items) for increasing revenue strategy. Conservative financial policy assures the safety of firms. Employing this policy, they do not face the potential liquidity issues, but profit will be reduced, because using too much long-term debts will increase the cost of capital rather than the use of short-term debt.

\section{Aggressive financial policy:}

Firms use short-term funds to finance the entire current assets and a portion of longterm assets. Employing this policy, they may face high risk due to rolling loans as well as increasing interest rates. However, short term interest rates is often lower than that of longterm loans, some companies are willing to sacrifice for a chance to raise higher profits. According to Eugene and Joel (2007), the reason for adopting a risky financial policy is the lower short-term rates (yield curve is 
usually slopes upward). However, strategy of financing for long-term assets by source shortterm capital really brings risk to firms. In some cases, firms may face temporary financial problems that they cannot pay the short-term liabilities coming due, creditors and lenders may refuse to extend their maturity leading to bankruptcy. Briefly, risky financing policy will generate a higher level of profitability, but also increases payment risk as well.

\section{Moderate financial policy:}

With this financing policy, fixed assets and a portion of permanent current assets are financed by long-term capital including longterm liabilities and owners' equity, and a part of permanent current assets is financed by short-term liabilities. This policy falls between the two extremes of aggressive policy and conservative policy so it brings about moderate risk and profitability comparing to the two other financial policies.

\subsection{Working capital management and firm's performance}

There are many different approaches on working capital management issues clarified by Ngo, T and Le (2015). Previous studies in different countries including countries in Asia, Europe, and Africa have shown that working capital management is the governance of current assets' components through the establishment and implementation of working capital management policies. The level of investment in current assets is the key basis for the effect of working capital management on firm performance. Excessive investment in short-term assets has a negative impact on firm efficiency. Conversely, limited investment in current assets may increase firm risk due to the probability of solvency. Working capital management is measured by cash conversion cycle. The cash conversion cycle is shortened leading to increase firm's profitability, in turn increase the firm value. Moreover, the shorter firm's cash conversion cycle, the higher net operating cash flows to firm from its operations.

Ngo, $\mathrm{T}$ and Le (2015) pointed out the close relationship between working capital management and firm performance. The research confirmed that the existence of the relationship between net operating profit and average inventory days, account payable days, and cash conversion cycle. Firms' cash conversion cycle and its components shorten will increase operating profits of the firms. The efficiency of working capital management not only increases profitability but also increases corporate market value.

For conservative financing policy, firm managers tend to maintain high cash holding and inventory level that may gain revenue growth, and reduce the shortage costs, in turn contributes to firm performance; however the policy may increase firm' financial costs and inventory carry costs that may affect adversely to firm performance, versus aggressive financing policy.

Table 1 summarizes the impact of working capital management on firm's performance measured by return on assets (ROA), and firm value measured by Tobin'Q. 


\section{Table 1}

Summary of previous research's result

\begin{tabular}{|c|c|c|c|c|c|}
\hline & Independent variable & Sign & & Authors & Method \\
\hline \multicolumn{6}{|c|}{ The impact of working capital management on ROA } \\
\hline \multirow[t]{2}{*}{1} & \multirow[t]{2}{*}{ Cash Conversion Cycle } & \multirow[t]{2}{*}{$\mathrm{CCC}$} & + & $\begin{array}{l}\text { Abuzayed (2011); Arbidance \& Ignatjeva (2012); } \\
\text { Ajanthan (2013); Asif \& Wang (2015) }\end{array}$ & $\begin{array}{l}\text { OLS ; FEM } \\
\text { REM; GMM }\end{array}$ \\
\hline & & & & $\begin{array}{l}\text { Garcia-Teruel \& Martinez Solano (2003); Deloof } \\
(2003) ; \text { Tryfonidis \& Lazaridis (2010); Gacia } \\
(2010) \text {; Sial \& Chauhdry (2010); Dong \& Su } \\
(2010) \text {; Ashraf (2012); Usama (2012); Mansoori \& } \\
\text { Muhammad (2012); Enqvist et al. (2012); } \\
\text { Ogundipe et al. (2012); Nobanee et al. (2012); } \\
\text { Nyamweno \& Olweny (2014) }\end{array}$ & $\begin{array}{l}\text { OLS ; FEM } \\
\text { GLS ; GMM }\end{array}$ \\
\hline \multirow[t]{2}{*}{2} & \multirow[t]{2}{*}{ Account Receivable Days } & \multirow[t]{2}{*}{ ARD } & + & $\begin{array}{l}\text { Abuzayed (2011); Arbidance \& Ignatjeva (2012); } \\
\text { Ajanthan (2013); Asif \& Wang (2015) }\end{array}$ & $\begin{array}{l}\text { OLS ; FEM } \\
\text { REM; GMM }\end{array}$ \\
\hline & & & & $\begin{array}{l}\text { Garcia-Teruel \& Martinez-Solano (2003) ; Gacia } \\
\text { (2010); Tryfonidis \& Lazaridis (2010) ; Dong \& Su } \\
\text { (2010); Sial \& Chauhdry (2010); Ashraf (2012); } \\
\text { Usama (2012); Mansoori \& Muhammad (2012); } \\
\text { Nyamweno \& Olweny (2014); Vương Đức Hoàng } \\
\text { Quân et al (2014) }\end{array}$ & $\begin{array}{l}\text { OLS ; FEM } \\
\text { GLS }\end{array}$ \\
\hline \multirow[t]{2}{*}{3} & \multirow[t]{2}{*}{ Average Inventory Days } & \multirow[t]{2}{*}{ AID } & + & $\begin{array}{l}\text { Abuzayed (2011); Arbidance \& Ignatjeva (2012); } \\
\text { Nyamweno \& Olweny (2014) }\end{array}$ & $\begin{array}{l}\text { OLS ; FEM } \\
\text { REM; GMM }\end{array}$ \\
\hline & & & & $\begin{array}{l}\text { Garcia-Teruel \& Martinez-Solano (2003); Gacia } \\
(2011) \text {; Sial \& Chauhdry (2010); Dong \& Su } \\
(2010) \text {; Tryfonidis \& Lazaridis (2010); Ashraf } \\
(2012) \text {; Usama (2012); } \\
\text { Mansoori \& Muhammad (2012) ; Vương Đức Hoàng } \\
\text { Quân et al (2014) }\end{array}$ & $\begin{array}{l}\text { OLS ; FEM } \\
\text { GLS }\end{array}$ \\
\hline \multirow[t]{2}{*}{4} & \multirow[t]{2}{*}{ Account Payable Days } & \multirow[t]{2}{*}{ APD } & + & $\begin{array}{l}\text { Tryfonidis \& Lazaridis (2010) ; Dong \& Su (2010) ; } \\
\text { Aridance \& Ignatjeva (2012) ; Usama (2012) }\end{array}$ & $\begin{array}{l}\text { OLS ; FEM } \\
\text { REM; GMM }\end{array}$ \\
\hline & & & & $\begin{array}{l}\text { Garcia-Teruel \& Martinez Solano (2003) ; Gacia } \\
\text { (2010); Sial \& Chauhdry (2010); Abuzayed (2011); } \\
\text { Ashraf (2012); Mansoori \& Muhamad (2012); } \\
\text { Vương Đức Hoàng Quân et al. (2014) }\end{array}$ & $\begin{array}{c}\text { OLS, FEM } \\
\text { GLS, GMM }\end{array}$ \\
\hline \multirow[t]{2}{*}{5} & \multirow{2}{*}{$\begin{array}{l}\text { Working Capital } \\
\text { Investment Policy }\end{array}$} & \multirow{2}{*}{ WCIP } & + & Mohamad \& Saad (2010) & OLS \\
\hline & & & - & Vahid (2012); Ogundipe et al (2012) & OLS, FEM \\
\hline \multirow[t]{2}{*}{6} & \multirow{2}{*}{$\begin{array}{l}\text { Working Capital Financial } \\
\text { Policy }\end{array}$} & \multirow{2}{*}{ WCFP } & + & Ogundipe et al (2012) & OLS \\
\hline & & & - & Mohamad \& Saad (2010); Vahid (2012) & OLS, FEM \\
\hline \multicolumn{6}{|c|}{ The impact of working capital management on Tobin's Q } \\
\hline 1 & Cash Conversion Cycle & $\mathrm{CCC}$ & & $\begin{array}{l}\text { Mohamad \& Saad (2010); Abuzayed (2011); } \\
\text { Ogundipe et al (2012) }\end{array}$ & OLS, GMM \\
\hline 2 & Account Receivable Days & ARD & - & Abuzayed (2011) & OLS, GMM \\
\hline 3 & Average Inventory Days & AID & - & Abuzayed (2011) & OLS, GMM \\
\hline 4 & Account Payable Days & APD & + & Abuzayed (2011) & OLS, GMM \\
\hline \multirow[t]{2}{*}{5} & \multirow{2}{*}{$\begin{array}{l}\text { Working Capital } \\
\text { Investment Policy }\end{array}$} & \multirow{2}{*}{ WCIP } & + & Mohamad \& Saad (2010) & OLS \\
\hline & & & - & Vahid (2012); Ogundipe et al. (2012) & OLS \\
\hline \multirow[t]{2}{*}{6} & \multirow{2}{*}{$\begin{array}{l}\text { Working Capital Financial } \\
\text { Policy }\end{array}$} & \multirow[t]{2}{*}{ WCFP } & + & Ogundipe et al (2012) & OLS \\
\hline & & & - & Mohamad \& Saad (2010); Vahid (2012) & OLS \\
\hline
\end{tabular}

Source: Journal of Banking Technology 


\subsection{Hypotheses and research models}

Based on working capital management theory and previous researches' results the research, hypotheses were developed as follows:

$\mathrm{H}_{1}$ : Cash conversion cycle impacts significantly negatively on firm performance (ROA).

$\mathrm{H}_{2}$ : Account receivable days impact significantly negatively on firm performance (ROA).

$\mathrm{H}_{3}$ : Average inventory days impact significantly negatively on firm performance (ROA).

$\mathrm{H}_{4}$ : Account payable days impact significantly positively on firm performance (ROA).

$\mathrm{H}_{5}$ : Cash conversion cycle impacts significantly negatively on firm value (Tobin'Q).

$\mathrm{H}_{6}$ : Account receivable days impact significantly negatively on firm value (Tobin'Q).

$\mathrm{H}_{7}$ : Average inventory days impact significantly negatively on firm value (Tobin'Q).

$\mathrm{H}_{8}$ : Account payable days impact significantly positively on firm value (Tobin'Q).

$\mathrm{H}_{9}$ : Working capital investment policy has a significant correlation with firm performance (ROA).

$\mathrm{H}_{10}$ : Working capital financing policy has a significant correlation with firm performance (ROA).
$\mathrm{H}_{11}$ : Working capital investment policy has a significant correlation with firm value (Tobin'Q).

$\mathrm{H}_{12}$ : Working capital financing policy has a significant correlation with firm value (Tobin'Q).

In order to test these hypotheses, eight research models were developed as below:

\section{Research model:}

ROAit $=\beta_{0}+\beta_{1}$ CCCit $+\beta_{2}$ WCIPit + $\beta_{3}$ WCFPit $+\beta_{4}$ SIZEit $+\beta_{5}$ CRit $+\beta_{6}$ DRit $+\beta_{7}$ EXPit + uit (1)

ROAit $=\beta_{0}+\beta_{1}$ ARDit $+\beta_{2}$ WCIPit + $\beta_{3}$ WCFPit $+\beta_{4}$ SIZEit $+\beta_{5}$ CRit $+\beta_{6}$ DRit $+\beta_{7}$ EXPit + uit (2)

ROAit $=\beta_{0}+\beta_{1}$ AIDit $+\beta_{2}$ WCIPit + $\beta_{3}$ WCFPit $+\beta_{4}$ SIZEit $+\beta_{5}$ CRit $+\beta_{6}$ DRit $+\beta_{7}$ EXPit + uit (3)

ROAit $=\beta_{0}+\beta_{1}$ APDit $+\beta_{2}$ WCIPit + $\beta_{3}$ WCFPit $+\beta_{4}$ SIZEit $+\beta_{5}$ CRit $+\beta_{6}$ DRit $+\beta_{7}$ EXPit + uit (4)

Tobin'Qit $=\beta_{0}+\beta_{1}$ CCCit $+\beta_{2}$ WCIPit + $\beta_{3}$ WCFPit $+\beta_{4}$ SIZEit $+\beta_{5}$ CRit $+\beta_{6}$ DRit $+\beta_{7}$ EXPit + uit (5)

Tobin'Qit $=\beta_{0}+\beta_{1}$ ARDit $+\beta_{2}$ WCIPit + $\beta_{3}$ WCFPit $+\beta_{4}$ SIZEit $+\beta_{5}$ CRit $+\beta_{6}$ DRit $+\beta_{7}$ EXPit + uit (6)

Tobin'Qit $=\beta_{0}+\beta_{1}$ AIDit $+\beta_{2}$ WCIPit + $\beta_{3}$ WCFPit $+\beta_{4}$ SIZEit $+\beta_{5}$ CRit $+\beta_{6}$ DRit $+\beta_{7}$ EXPit + uit (7)

Tobin'Qit $=\beta_{0}+\beta_{1}$ APDit $+\beta_{2}$ WCIPit + $\beta_{3}$ WCFPit $+\beta_{4}$ SIZEit $+\beta_{5}$ CRit $+\beta_{6}$ DRit $+\beta_{7}$ EXPit + uit (8)

The variables including in the research models are determined as shown in Table 2. 


\section{Table 2}

Variables description

\begin{tabular}{|c|c|c|c|c|}
\hline & Notation & Variable name & Calculation & Sign \\
\hline \multicolumn{5}{|c|}{ Dependent variables } \\
\hline 1 & ROA & Returns on assets & Earning before tax/Total assets & \\
\hline 2 & Tobin'Q & Firm's value & $\begin{array}{l}\text { (Market value of owner equity }+ \text { net book } \\
\text { value of liabilities)/ (Net book value of owner } \\
\text { equity }+ \text { net book value of liabilities) }\end{array}$ & \\
\hline
\end{tabular}

\section{Main independent variables}

1 CCC Cash Conversion cycle

2 ARD Account Receivable days

3 AID Average Inventory Days

4 APD Account Payable Days

5 WCIP Working Capital Investment Policy

6 WCFP Working Capital Financial Policy
Account Receivable Days + Average Inventory Days - Account Payable Days

(Average Account receivable x 365)/revenue

(Average inventory x 365)/Cost of goods sold

(Average account payable x 365)/purchase

Total current assets/Total asset

Total current- liabilities/Total asset
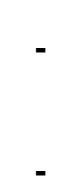

$-$

$+$

$-$

$+$

\section{Control variables}

7 SIZE Firm's scale

8 CR Current Ratio

9 DR Debt Ratio

10 EXP Exported Proportion
Logarithm of total asset

Total current-asset/Total current liabilities

Total liabilities/Total asset

Total exported revenues/Total Revenue

\section{Method}

Quantitative method with panel data was used to test the hypotheses in the study. Data was collected from the financial statements of 21 fisheries companies listing on Vietnam stock markets (HOSE and HNX) from 2008 2012. There are 105 observations presented in panel data.

Multivariate regression analysis with Generalized Method of Moment (GMM) developed by Hansen'J (1982) was conducted in Stata software version 12. According to Baum et al (2003), GMM regression estimation method produces consistant and effective estimator and overcomes the assumptions of linear regression model such as heterokadasticity, autocorrelation and endogeneity. It is not worse than the other estimation methods. However, in order to have an efficient GMM estimation, two conditions need to be satisfied. The first condition is the highly correlated between independent variables with the endogenous variable. The second is independent variables which aren't correlated with the residual (Baum, 2003; Guikey, 2004; Bond, 2007; Fisher, 2010). Research has also done the necessary testing 
to ensure these conditions.

\section{Findings}

\subsection{Statistic description}

Table 3 describes statistics of variables in the research models. For fisheries firms in the period from 2008 to 2012, in average their cash conversion cycle around 137 days, average collection period around 38 days, days sales in inventory around 104 days, payables days around 25 days.

\section{Table 3}

Descriptive statistic of variables

\begin{tabular}{llccccccc}
\hline \multicolumn{1}{c}{ Variable name } & Code & Mean & Median & $\begin{array}{c}\text { Standard } \\
\text { Deviation }\end{array}$ & Kurtos-is & $\begin{array}{c}\text { Skews } \\
\text {-ness }\end{array}$ & Min & Max \\
\hline Returns On Assets & ROA & 0,06 & 0,05 & 0,02 & 1,08 & 0,14 & $-0,16$ & 0,24 \\
Firm's value & TOBIN'Q & 1,01 & 0,98 & 0,23 & 1,45 & 0,99 & 0,61 & 1,83 \\
Cash Conversion Cycle & CCC & 137,45 & 118,96 & 10,86 & 36,88 & 5,00 & 15,84 & 994,82 \\
Account Receivable Days & ARD & 58,28 & 46,28 & 36,27 & 2,61 & 1,38 & 10,63 & 217,09 \\
Average Inventory Days & AID & 104,31 & 79,14 & 32,09 & 34,08 & 4,91 & 31,56 & 814,69 \\
Account Payable Days & APD & 25,14 & 20,84 & 12,14 & 5,32 & 1,72 & 10,86 & 109,88 \\
Working Capital Investment & WCIP & 0,67 & 0,69 & 0,11 & $-0,25$ & $-0,60$ & 0,38 & 0,89 \\
Policy & & & & & & & & \\
Working Capital Financial & WCFP & 0,51 & 0,54 & 0,20 & $-0,60$ & $-0,38$ & 0,06 & 0,89 \\
Policy & & & & & & & & \\
Firm's scale & SIZE & 1008,5 & 552,6 & 1272,8 & 10,04 & 2,59 & 39,3 & 6365,9 \\
Current Ratio & CR & 1,77 & 1,19 & 1,14 & 16,70 & 3,83 & 0,63 & 10,37 \\
Debt Ratio & DR & 0,57 & 0,59 & 0,21 & $-0,40$ & $-0,59$ & 0,06 & 0,93 \\
Exported Proportion & EXP & 0,79 & 0,81 & 0,22 & 37,70 & 4,52 & 0,30 & 0,98 \\
\hline
\end{tabular}

Source: make by authors and information is extracted from software Stata 12

Most of the fisheries firms have returns of assets (ROA) around 6\%. The value of Tobin'Q is 1.01 , or the market value of selected fisheries companies is higher than their investment value, however, the difference is not too much.

Cash conversion cycle has mean value greater than 137 days and the median is 118 days, and the skewness factor is 5 bigger than 1 (beyond the range $\{-1: 1\})$. We can conclude that most of selected fisheries firms have cash conversion cycle (CCC) less than 137 days. Similarly, account receivable days (ARD) of most selected companies is less than the average of 58 days; their average inventory days (AID) is less than 104 days, account payable days (APD) mostly less than 25 days.

Current assets to total asset ratio, the representative of working capital investment policy (WCIP) is 0.67 showing that most of selected fisheries companies have invested heavily in their current assets, adopting the conservative working capital investment policy. In addition, working capital financial policy (WCFP) measured by the ratio of current liabilities to total assets has the average value of 0.51 . In other words, almost current assets of selected fisheries firms are financed by short term liabilities. 


\subsection{Hypothesis testing}

To test research hypotheses $\mathrm{H}_{1}, \mathrm{H}_{2}, \mathrm{H}_{3}$, $\mathrm{H}_{4}, \mathrm{H}_{9}$ and $\mathrm{H}_{10}$, GMM estimation method was used and the test's results are presented in Table 4. The results showed that cash conversion cycle (CCC), average receivable days (ARD), and average inventory days (AID), and average payable days (APD) impact negatively on ROA at the significance level of $1 \%$, or $\mathrm{H}_{1}, \mathrm{H}_{2}, \mathrm{H}_{4}$ are accepted.
Hypothesis 3 proposed APD impacts positively on ROA, however the test resulted the opposite sign. Working capital investment policy (WCIP) impacts positively on ROA at $1 \%$ significant level, while working capital financial policy (WCFP) affects negatively to ROA at $1 \%$ and $10 \%$ significant level. Thus, $\mathrm{H}_{9}$ and $\mathrm{H}_{10}$ are accepted. Control variables have no impact on ROA excepting current ratio.

\section{Table 4}

The testing results for Hypotheses 1, 2, 3, 4, 9, and 10

\begin{tabular}{|c|c|c|c|c|}
\hline \multicolumn{5}{|c|}{ Dependent variable : ROA } \\
\hline VARIABLE & MODEL 1 & MODEL 2 & MODEL 3 & MODEL 4 \\
\hline $\mathrm{CCC}$ & $-0.00018 * * *$ & & & \\
\hline ARD & & $-0.00075^{* * *}$ & & \\
\hline AID & & & $-0.00020 * * *$ & \\
\hline APD & & & & $-0.00292 * * *$ \\
\hline WCIP & $0.34265 * * *$ & $0.32928 * * *$ & $0.33337 * * *$ & $0.23118 * * *$ \\
\hline WCFP & $-0.28664 * * *$ & $-0.30228 * * *$ & $-0.26564 * * *$ & $-0.11918 *$ \\
\hline SIZE & -0.01537 & -0.00535 & -0.01846 & -0.00383 \\
\hline $\mathrm{CR}$ & $-0.01101 * * *$ & $-0.01151 * * *$ & $-0.00991 * *$ & $-0.00182 *$ \\
\hline EXP & -0.04720 & -0.04697 & -0.04556 & -0.05096 \\
\hline Constant & 0.14996 & 0.12917 & 0.15576 & 0.10762 \\
\hline $\mathrm{R} 2$ & 0.3968 & 0.3857 & 0.3865 & 0.2215 \\
\hline Statistic F & $76.54 * * *$ & $73.82 * * *$ & $77.51 * * *$ & $75.41 * * *$ \\
\hline Observations & 105 & 105 & 105 & 105 \\
\hline $\begin{array}{l}\text { Hansen'J } \\
\text { (Overidentifying) }\end{array}$ & 0.2175 & 0.1673 & 0.7758 & 2.9803 \\
\hline $\mathrm{P}$ - value & 0.6409 & 0.6876 & 0.3784 & 0.2253 \\
\hline $\begin{array}{l}\text { Wu-Hausman } \\
\text { (Orthogonality) }\end{array}$ & 3.8871 & 4.3322 & 5.0150 & 8.6004 \\
\hline $\mathrm{P}$-value & 0.0487 & 0.0374 & 0.0251 & 0.0034 \\
\hline $\begin{array}{l}\text { First stage R2 } \\
\text { Partial (IV) }\end{array}$ & 0.7021 & 0.3781 & 0.7654 & 0.3346 \\
\hline Statistic F (IV) & $19.67 * * *$ & $16.02 * * *$ & $22.31 * * *$ & $9.36 * * *$ \\
\hline
\end{tabular}

Source: make by authors and information is extracted from software Stata 12

Notice: * level of significance $10 \%$; ** level of significance $5 \%$, *** level of significance $1 \%$ 
To test research hypotheses $\mathrm{H}_{5}, \mathrm{H}_{6}, \mathrm{H}_{7}$, $\mathrm{H}_{8}, \mathrm{H}_{11}$ and $\mathrm{H}_{12}$, GMM estimation method was used and the test's results are presented in Table 5. The results showed that cash conversion cycle (CCC), average receivable days (ARD), and average inventory days (AID), and average payable days (APD) impact negatively on Tobin'Q at the significance level of $1 \%$ and $5 \%$, or $\mathrm{H}_{5}, \mathrm{H}_{6}$, $\mathrm{H}_{8}$ are accepted. Hypothesis 7 proposed APD impacts positively on Tobin'Q, however the test resulted the significantly negative impact. Working capital investment policy (WCIP) has no impact on Tobin'Q (Model 5, 6, and 7) excepting Model 8, in which it impacts on Tobin'Q positively at 5\% significant level. Working capital financial policy (WCFP) has no a significant influence on Tobin'Q (Model 5, 6, and 7), but has a positive relationship with Tobin'Q at 5\% significant level (Model 8). Thus, $\mathrm{H}_{11}$ and $\mathrm{H}_{12}$ are partial accepted.

\section{Table 5}

The testing results for Hypotheses 5, 6, 7, 8, 11 and 12

\begin{tabular}{|c|c|c|c|c|}
\hline \multicolumn{5}{|c|}{ Dependent variable : TOBIN'Q } \\
\hline VARIABLE & MODEL 5 & MODEL 6 & MODEL 7 & MODEL 8 \\
\hline $\mathrm{CCC}$ & $-0.00061 * * *$ & & & \\
\hline ARD & & $-0.00105^{* * *}$ & & \\
\hline AID & & & $-0.00079 * * *$ & \\
\hline APD & & & & $-0.00106^{* *}$ \\
\hline WCIP & 0.10670 & 0.02611 & 0.04769 & $0.46571 * *$ \\
\hline WCFP & $-0.20112^{* * *}$ & $-0.21109 * * *$ & $-0.14949 * *$ & $-0.38801 *$ \\
\hline SIZE & -0.04744 & -0.01279 & -0.04401 & -0.03429 \\
\hline $\mathrm{CR}$ & $-0.03360^{* * *}$ & $-0.02954 * * *$ & $-0.03169 * * *$ & -0.02121 \\
\hline EXP & -0.05156 & -0.02541 & -0.01095 & -0.6626 \\
\hline L.TOBINQ & $0.62871 * * *$ & $0.65481 * * *$ & $0.61943 * * *$ & $0.71366^{* * *}$ \\
\hline Constant & 0.82481 & 0.60668 & 0.78167 & 0.46861 \\
\hline $\mathrm{R} 2$ & 0.7027 & 0.7420 & 0.6751 & 0.3348 \\
\hline Statistic F & $354.25^{* * *}$ & $389.97 * * *$ & $291.04 * * *$ & $146.27 * * *$ \\
\hline Observations & 84 & 84 & 84 & 84 \\
\hline Hansen’J (Overidentifying) & 4.2261 & 0.0001 & 0.7466 & 0.0029 \\
\hline P-value & 0.1209 & 0.9967 & 0.3875 & 0.9565 \\
\hline Wu-Hausman (Orthogonality) & 4.8048 & 3.9835 & 10.7867 & 15.8084 \\
\hline P-value & 0.0284 & 0.0679 & 0.0010 & 0.0001 \\
\hline First stage R2 Partial (IV) & 0.3762 & 0.5115 & 0.3112 & 0.3296 \\
\hline Statistic F (IV) & $15.71 * * *$ & $28.85 * * *$ & $10.97 * * *$ & $9.93 * * *$ \\
\hline
\end{tabular}

Source: make by authors and information is calculated by software Stata 12

Notice: * level of significance $10 \%$; ** level of significance $5 \%$, *** level of significance $1 \%$ 


\section{Discussion}

- The effect of cash conversion cycle to firm performance (ROA) and firm value (Tobin'Q) of selected fisheries companies.

Cash conversion cycle has a negative correlation with return on assets (ROA) and Tobin'Q). The result implies that the longer cash conversion cycle, the lower ROA of selected fisheries firms is. Thus, shortening the cash conversion cycle will increase cash available to use and increase company's liquidity, in turn increase firm profitability and firm value. The finding is consistent with the findings of Ashraf (2012); Mansoori and Muhammad (2012); Enqvist et al. (2012); Ogundipe et al. (2012); and Nobanee et al. (2012).

- The effect of account receivable days (ARD) to firm performance (ROA) and firm value (Tobin' $Q$ ) of selected fisheries firms.

The account receivable days (ARD) has a negative correlation with return on assets (ROA) and firm's value (Tobin'Q). The shorter account receivable days reduces the firm's cash conversion cycle, and then increases the firm performance and its value. The finding is consistent with the finding of Ashraf (2012); Usama (2012); and Mansoori $\&$ Muhammad (2012).

- The impact of average inventory days (AID) to firm performance (ROA) and firm value (Tobin'Q) of selected fisheries companies.

Fisheries firms often have the high shortage level of inventory because of their seasonal nature. The storage of inventory for production and selling helps fisheries firm avoid negative impact of price fluctuation caused by commodity scarce. However, a firm stores inventory at a high level will be burdened carry expenses (Ashraf, 2012) resulting reducing firm profitability and value. The finding is consistent with that of Ashraf (2012); Usama (2012); and Mansoori and Muhammad (2012).
- The impact of account payable days (APD) to firm performance (ROA) and firm value (Tobin'Q) of selected fisheries firms.

In fact, firms often purchase materials and goods on account, or accept trade credit provided by their suppliers. Typically, to minimize cash balance holding, firms try to delay the payment as long as possible. However, the cost of trade credit is usually higher than short-term loan interest rate, so stretching the payment for vendor in the long run will break the business relationship and then reduces firm value (Mansoori and Muhammad, 2012). The excesses of benefit gain to cost incurred will reduce firm performance and value.

- The impact of working capital investment policy (WCIP) to firm performance (ROA) and firm value (Tobin'Q) of selected fisheries firms.

The research result from regression models does not provide empirical evidence of the relationship between working capital investment policy and Tobin'Q of selected fisheries firms. However, the research provides the evidence that the high level of investment in current assets, the high return on assets of selected fisheries firms.

- The impact of working capital financial policy (WCFP) to firm performance (ROA) and firm value (Tobin'Q) of selected fisheries firms.

The study provides the evidence that increasing the level of short-term debt to finance current assets will reduce firm performance (ROA), and firm value (Tobin'Q), which is consistent with the findings of Vahid (2012); and Mohamad and Saad (2010).

\section{Conclusion and Recommendation}

The research conducted on 21 fisheries firms listing on HOSE and HNX provided the negative effects of cash conversion cycle, average inventory days, account receivable days and account payable days to ROA and 
Tobin'Q of selected fisheries firms. Moreover, the study also indicated that there is a negative correlation between aggressive working capital financial policy and ROA. On another hand, there is a positive correlation between conservative working capital financial policy and ROA. From this conclusion, the study also recommends some management policies of working capital in fisheries sector that should be considered.

The research findings imply that fisheries firms should reduce cash conversion cycle, average inventory days, account receivables days and account payable days, and should apply conservative working capital investment policy to increase firm performance (ROA). Doing that, firms need to push up the production process and improve selling process, collection policy as well.

For working capital financial policy, fisheries firms should finance current assets by long term funds to improve firm performance.

Since the days purchase in account payable has a negative relationship with firm performance, firms should select suppliers providing loosing credit policy, or manage payment well

\section{References}

Ashraf, C.K. (2012). The relationship between working capital eficiency and profittability. Department of Business and Financial Studies, University of Kashmir, 5(12), 60-74.

Enqvist, J., Graham, M. và Nikkinen, J. (2012). The impact of working capital management on firm profitability in different business cycles: Evidence from Finland. Social Science Research Network, http://www.ssrn.com.

Firer, O. và Ajilore, O. (2009). Working capital management and coporate profittability: Evidence from Panel data analysis of selected quoted companies in Nigieria. Journal of Business Manageent, 3, 73-84.

Mansoori, E.M. và Muhammad, D.J. (2012). The effect of working capital management on firm's profitability: Singapore. Interdisciplinary Journal of Contemporary Research in Business, 4(5), 472-486.

Mohamad, A.B và Saad, B.M. (2010). Working capital management: The effect of market valuation and profitability in Malaysia. International Journal of Business and Management, 5(11), DOI: 10.5539/ijbm.v5n11p140.

Nobanee, H. và Al Hajjar, M. (2011). Cash conversation cycle and firm's performance of Japanese firms. Emerald Article, 19(2), 147-156.

Ogundipe, E., Sunday, A.I. và Ogundipe, O.L. (2012). Working capital management, Firm's performance and market valuation in Nigeria. International Journal of Social and Human Sciences, 2, 1196-1200.

Usama, M. (2012). Working capital management and its effect on firm's profitability and liquidity: In other sector of Karachi Stock Exchange. Arabian of Business and Management Review, 1(12), 62-73.

Vahid, T.K., Mohsen, A.K. và Mohammadreza, E. (2012). The impact of working capital management policies on firm's profitability and value: Evidence from Iranian companies. International Research Journal of Finance and Economics, 88, 1450-2087.

Ngo, T and Le, N (2015). An overview of researches regarding to the impact of working capital management on firm's performance. Banking Technology Review, 109, 55. 